\title{
Inferior olive CRF plays a role in motor performance under challenging conditions
}

\author{
Gili Ezra-Nevo ${ }^{1,2}$, Naama Volk ${ }^{1,2}$, Assaf Ramot ${ }^{1,2}$, Claudia Kuehne ${ }^{2}$, Michael Tsoory ${ }^{3}$, Jan Deussing (1) ${ }^{2}$ and Alon Chen (i) ${ }^{1,2}$
}

\begin{abstract}
A well-coordinated stress response is pivotal for an organisms' survival. Corticotropin-releasing factor (CRF) is an essential component of the emotional and neuroendocrine stress response, however its role in cerebellar functions is poorly understood. Here, we explore the role of CRF in the inferior olive $(I O)$ nucleus, which is a major source of input to the cerebellum. Using a CRF reporter line, in situ hybridization and immunohistochemistry, we demonstrate very high levels of the CRF neuropeptide expression throughout the $1 \mathrm{O}$ sub-regions. By generating and characterizing IOspecific CRF knockdown and partial IO-CRF knockout, we demonstrate that reduction in IO-CRF levels is sufficient to induce motor deficiency under challenging conditions, irrespective of basal locomotion or anxiety-like behavior.

Furthermore, we show that chronic social defeat stress induces a persistent decrease in IO-CRF levels, and that IO-CRF mRNA is upregulated shortly following stressful situations that demand a complex motor response. Taken together our results indicate a role for IO-CRF in challenge-induced motor responses.
\end{abstract}

\section{Introduction}

When a situation is perceived as stressful, the brain activates many neuronal circuits, linking centers involved in sensory, motor, neuroendocrine, cognitive, and emotional functions in order to adapt to the demand. There is substantial evidence to suggest that inappropriate regulation, disproportional intensity, or chronic and/or irreversible activation of the stress response is linked to the etiology and pathophysiology of anxiety and depression ${ }^{1-}$ 5 .

Corticotropin-releasing factor (CRF) and its type 1 receptor (CRFR1) have a unique role in mediating behavioral and physiological responses to diverse stressors. These systems may be particularly important in situations where an organism must mobilize not only the hypothalamic-pituitary-adrenal (HPA) system, but also the central nervous system in response to environmental

Correspondence: Alon Chen (alon.chen@weizmann.ac.il)

'Department of Neurobiology, Weizmann Institute of Science, Rehovot 76100, Israel

${ }^{2}$ Department of Stress Neurobiology and Neurogenetics, Max Planck Institute of Psychiatry, 80804 Munich, Germany

Full list of author information is available at the end of the article challenge ${ }^{1,2,5}$. Interestingly, it has been repeatedly demonstrated that CRF is prominently expressed in the inferior olive (IO) of various species from rodents to primates, as well as localized in the fibers descending from the IO, namely, the climbing fibers $(\mathrm{CFs})^{6-17}$.

The IO, which constitutes one of the two major inputs to the cerebellum and to Purkinje cells (PCs) in particular, is best known for its role in motor adjustment, coordination, balance, and learning of motor skills ${ }^{18,19}$. Interestingly, altered cerebellar connectivity and vestibular problems were found in PTSD patients and several forms of anxiety disorders, however the interaction between acute or chronic stress and motor performance is still poorly understood ${ }^{20-23}$.

In this study, we explored the contribution of CRF in the IO to the well-orchestrated stress response of the adult mouse. We show that site specific reduction of IOCRF in the adult mouse was sufficient to induce a challenge-induced motor deficit, without affecting baseline motor activity. We show that chronic social defeat stress (CSDS) induces a persistent decrease in IO-CRF levels. We further present evidence that IO-CRF is regulated following specific stressors that comprise a motor

\section{(c) The Author(s) 2018}

(c) (i) Open Access This article is licensed under a Creative Commons Attribution 4.0 International License, which permits use, sharing, adaptation, distribution and reproduction cc) in any medium or format, as long as you give appropriate credit to the original author(s) and the source, provide a link to the Creative Commons license, and indicate if changes were made. The images or other third party material in this article are included in the article's Creative Commons license, unless indicated otherwise in a credit line to the material. If material is not included in the article's Creative Commons license and your intended use is not permitted by statutory regulation or exceeds the permitted use, you will need to obtain permission directly from the copyright holder. To view a copy of this license, visit http://creativecommons.org/licenses/by/4.0/. 
challenge indicating CRF "participation" in coping with these challenges.

\section{Materials and methods}

Animals and experimental groups

Detailed description of the animals and experimental groups used in this study can be found in SI Methods.

\section{Behavioral studies}

All behavioral paradigms were performed as previously described $^{24-26}$ with modifications. For a detailed description of the behavioral tests, see SI Methods.

\section{Immunohistochemistry and in situ hybridization}

In situ hybridization ${ }^{27-29}$ and immunohistochemistry $^{26,30}$ were performed as described. For a full description of the protocols and antibodies used, see SI Methods.

\section{CLARITY and whole-brain imaging}

The CLARITY method used here was based on protocols reported by Ye et al. ${ }^{31}$ and modified. A detailed description can be found in the SI Methods.

\section{Viral constructs and stereotaxic injections}

All constructs were assembled by using standard cloning methods and confirmed by DNA sequencing and described in ${ }^{27,28,32}$. Stereotaxic injections were performed as described by Ramot et al. ${ }^{26}$. For a detailed description, see SI Methods.

\section{mRNA extraction and quantification with qRT-qPCR}

Preparation and quantification of mRNA was performed as previously described ${ }^{30}$. A full description can be found in SI Methods.

\section{Statistical analysis}

Results are expressed as mean \pm SEM. Automated analysis was used whenever possible, including cell counting and behavioral measurements. Samples that were 2 SD above or below the group mean were excluded. Statistical analysis was conducted using SPSS software (SPSS Inc, Chicago, IL). Following a test of normality, statistical significance was determined by Student's $t$-test or oneway analysis of variance (ANOVA) followed by Fisher's LSD post hoc test, or by non-parametric test (Mann-Whitney $U$ test; MW) or two-way repeated measures ANOVA, when appropriate. No differences in variance between groups were statistically significant. Statistical tests were two-sided. $p<0.05$ was considered significant.

\section{Results}

CRF is expressed throughout the $I O$ and highly expressed in both the mouse and human 10

We validated CRF expression and sub-nuclei distribution in the IO using the CRF-Cre mouse line ${ }^{33}$ crossbred with mice conditionally expressing tdTomato ${ }^{34}$ (CRFtdTomato; Fig. 1a-c, Supplementary Fig.1B), as well as in situ hybridization (ISH) for CRF mRNA (Fig. 1c, Supplementary Fig.1C). The tdTomato reporter signal produced by the CRF positive IO neurons was clearly evident throughout the IO and in the CF reaching the cerebellum, as seen in Fig. 1a, b, d, and more elaborately in Supplementary Fig.1A-C. Supplementary Movie 1 of a CRFtdTomato mouse brain imaging depicts the profuse tdTomato positive staining of fibers going from the IO to the cerebellum. CRF ISH shows a similar expression pattern (Fig. 1c). CFs originating in CRF expressing cells (showing tdTomato signal) reach the cerebellar cortex and climb over PCs (stained in Cyan; gray arrows indicate CFs in close proximity to PCs dendritic tree; Fig. 2d). We used colchicine injected mice to quantify the percentage of CRF positive neurons within the IO. Coronal brain sections from these mice were immunostained for both NeuN (green) and CRF (red; Fig. 1e, f) along with Hoechst staining (blue). This triple staining revealed that more than $70 \%$ of IO neurons express CRF (Fig. 1g). An additional assessment of the percentage of CRF-positive cells in the IO was performed using CRF-Ai9 mice with calbindin (an IO marker) IO immunostaining. This analysis yielded similar results, again indicating that $\sim 70 \%$ of IO neurons express CRF (Supplementary Fig. 1). Lastly, data of relative expression of CRF in humans, obtained from the Allen human brain atlas, shows CRF has an extremely high expression level in the human IO (Fig. 1h; Allen Brain institute, Human Microarray data, 2016, ${ }^{35}$; probe 1057965). These results indicate that IO-CRF is likely to play a conserved role in both the mouse and human cerebellar system.

\section{Intact home cage locomotion but impaired challenge- induced motor performance in IO-specific CRF knockdown mice}

We next examined whether reduced IO-CRF was sufficient to induce motor impairment. To that end, we injected lentivirus expressing previously validated shCRF (knockdown, KD) or control construct into the IO of adult C57/BL mice ${ }^{27,28}$; IO-CRF $\mathrm{KD}$; Fig. 2a). Quantification of the CRF mRNA signal using ISH (Fig. 2b, c) on brain slices showed a CRF mRNA signal reduction of $\sim 50 \%$ in the IO area of KD mice compared to controls ( $t$ test, $t_{(10)}=3.818, p=0.003$; Fig. 2d).

Mice injected with the $\mathrm{KD}$ virus into the $\mathrm{IO}\left(\mathrm{IO}-\mathrm{CRF}_{\mathrm{KD}}\right)$ did not differ in home-cage locomotion from controlinjected mice during the light or the dark phases (Fig. 2e, 


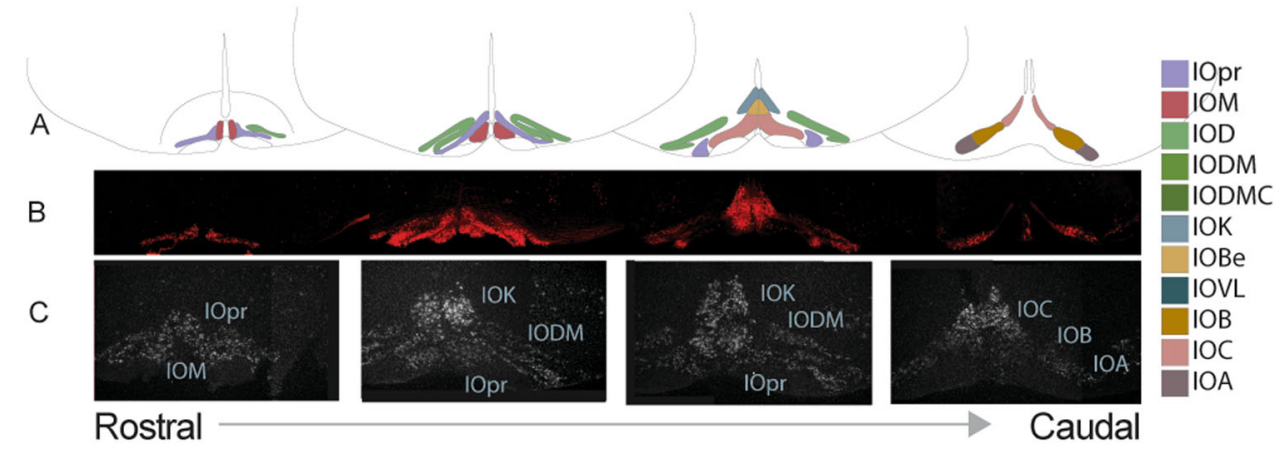

D
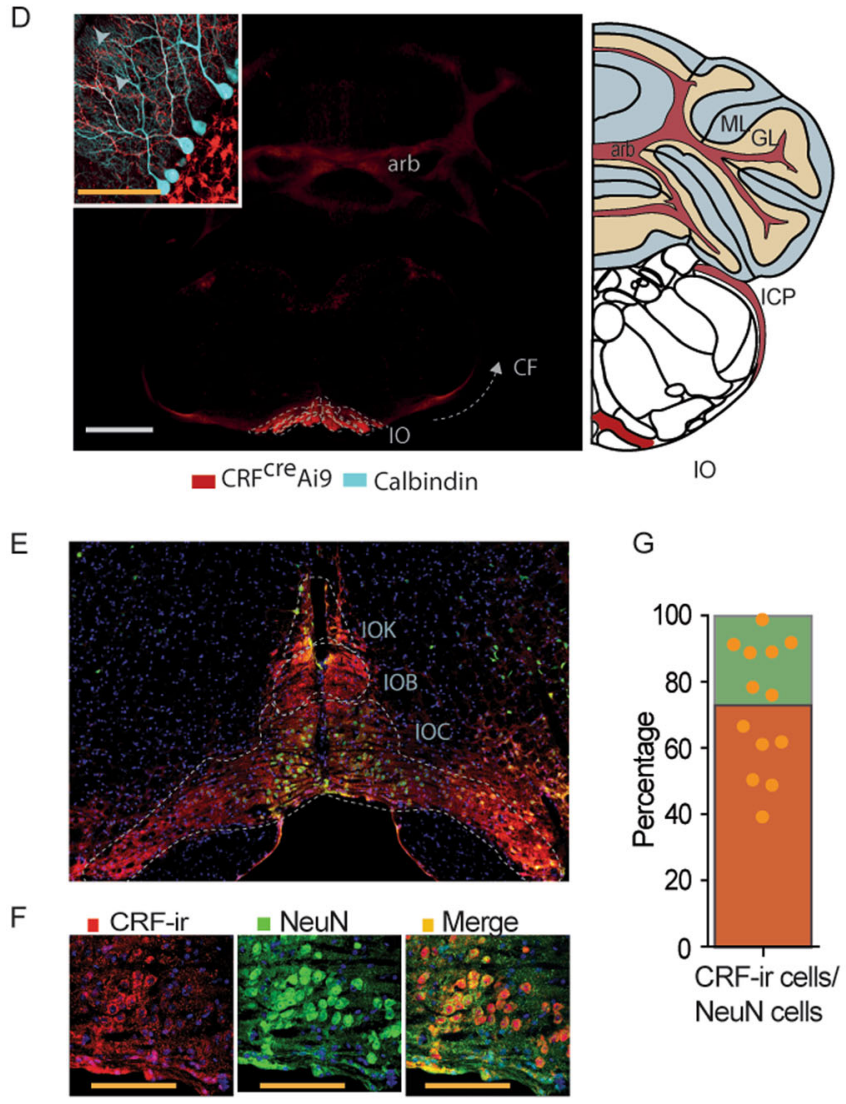

G

$\mathrm{H}$
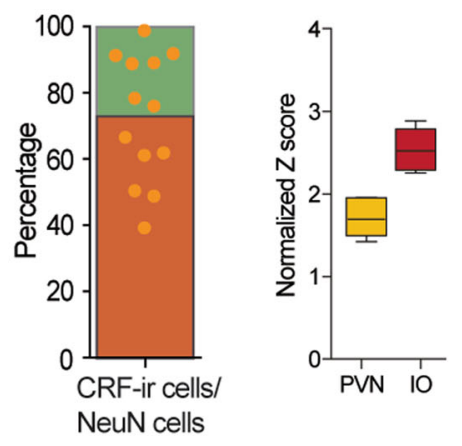

Fig. 1 CRF is highly expressed in the 10 of the mouse. a Schematic coronal representation of four slices of the 10 and its sub-nuclei. $1 O p r 10$ principal nucleus, IOD IO dorsal nucleus, IODM IO dorsomedial cell group, IOM IO medial nucleus, IODMC IO dorsomedial cell column, IOK cap of Kooy medial nucleus, IOBe IO beta subnucleus, IOV IO ventral nucleus, IOB IO subnucleus B medial nucleus, IOC IO subnucleus C medial nucleus, IOA IO subnucleus A medial nucleus. b The IO of a CRF-Cre mouse line crossbred with conditional tdTomato mouse line (CRF-tdTomato; red). tdTomato signal indicates CRF expression, which is seen from the most rostral part to the most caudal structures of the IO. c CRF In situ hybridization of wild type (WT) mouse. Dark field images show high CRF mRNA signal throughout the IO of the mouse, compatible with expression patterns seen in the reporter line. $\mathbf{d}$ Coronal section of a CRF-tdTomato mouse stained for calbindin (cyan), a Purkinje cell (PC) marker. Image depicts climbing fibers (CF) expressing tdTomato (red), indicating CRF expressing cells reach the molecular layer of the cerebellar cortex (gray arrows indicate tdTomato expressing fibers in close proximity to PCS dendritic tree). ICP inferior cerebellar peduncle, ML molecular layer, GL granular layer, arb arbor vitae, IOK cap of Kooy medial nucleus, IOB IO subnucleus B medial nucleus, IOC IO subnucleus C medial nucleus Gray scale bar $($ bottom) $=1000 \mu \mathrm{m}$, orange scale bar (top left) $=100 \mu \mathrm{m}$. e Representative image of mouse IO stained with CRF antibody (red) and neuronal marker (NeuN; green), along with Hoechst staining (blue). $\mathbf{f}$ Representative Hoechst stained (blue) images of $\mathrm{IO}$ cell bodies co-stained for CRF (red) and NeuN (green), and a merged image. Orange scale bar $=100 \mu \mathrm{m}$. g Summary of IO NeuN-positive cells stained for CRF. $70 \%$ of NeuN stained cells (green bar), are co-stained for CRF (orange bar; based on the staining of 6 and $7 \mathrm{IO}$ sections from 2 mice, a total of 13 sections, each dot in the graph represents an IO slice). $\mathbf{h}$ Relative quantity of CRF mRNA from humans obtained from the Allen brain institute for Brain Science. Data (from six donors) shows that in humans, CRF expression in the $\mathrm{IO}$ is even higher than in the paraventricular nucleus of the hypothalamus, the hallmark region for CRF expression 

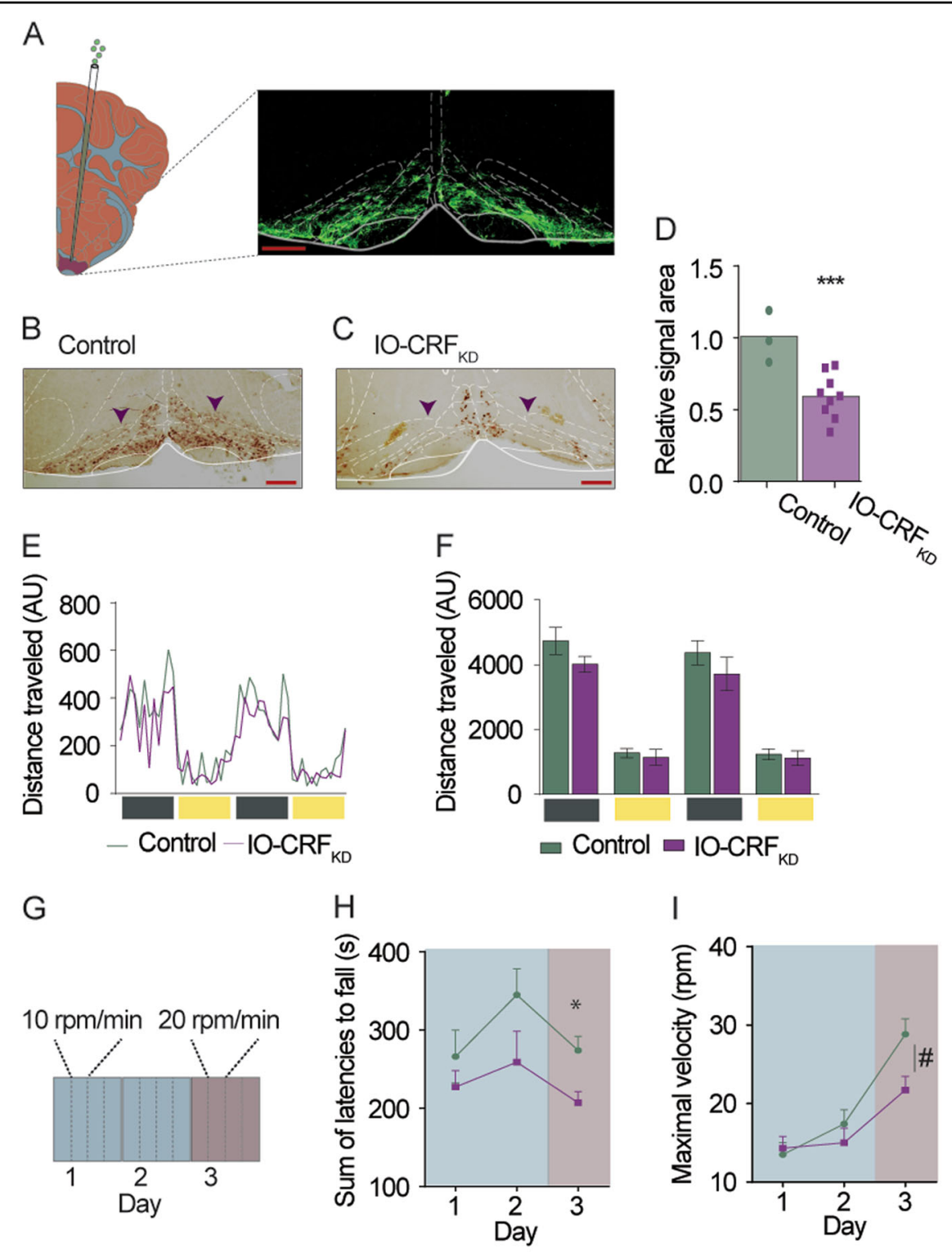

- Control $=1 \mathrm{O}-\mathrm{CRF}_{\mathrm{KD}}$

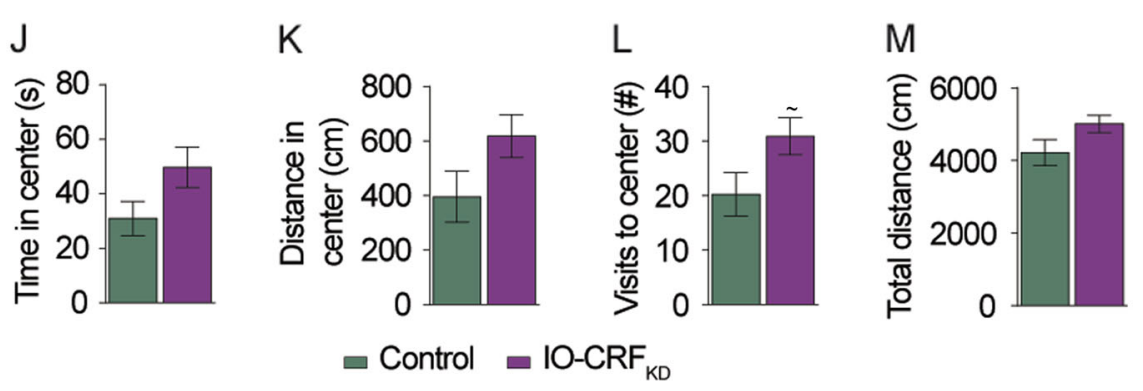

Fig. 2 (See legend on next page.) 
Fig. 2 Intact home cage locomotion but impaired challenge-induced motor performance in IO-specific CRF KD mice. Knockdown (KD) of CRF specifically in the $I O$ of adult mice is sufficient to impair motor performance on the rotarod. a Schematic illustration of lentiviral injection and a representative confocal microscope image of a virally infected IO of an adult C57 mouse. Red scale bar $=250 \mu \mathrm{m}$. In situ hybridization using probes for CRF showing the $\mathrm{IO}$ of mice injected with $\mathbf{b}$ control virus and $\mathbf{c} K D$ virus. Red scale bar $=250 \mu \mathrm{m}$. $\mathbf{d}$ Relative CRF in situ hybridization signal area of $\mathrm{KD}$ and control injected $\mathrm{IO}$ (each dot represents a slice, $n=3,9$ slices). e, $\mathbf{f}$ Baseline locomotion of IO-CRF $\mathrm{KD}_{\mathrm{K}}$ mice and control mice was measured using inframot. KD mice did not differ from control mice in home-cage locomotion $(n=7,9)$. $\mathbf{g}$ Schematic illustration of the rotarod protocol used. Mice underwent 2 days of training with the rotarod accelerating from 0 to $40 \mathrm{rpm}$ in $4 \mathrm{~min}$ (inclination of $10 \mathrm{rpm} / \mathrm{min}$ ) then on the $3 \mathrm{rd}$ day, the rotarod was accelerated from 0 to 40 in $2 \mathrm{~min}(20 \mathrm{rpm} / \mathrm{min})$. Each day, mice were subjected to four trials with a 2-min break in between. Sum of latencies to fall and maximal velocity reached were measured. IO-CRF $\mathrm{KD}$ and control mice motor performance was tested using the rotarod. $\mathbf{h}$ Although on average IO-CRF $\mathrm{KD}$ lasted less time on the rotarod compared to control mice, no overall significant differences were detected between the groups, but significant difference in latency to fall was seen at the highest velocity $(20 \mathrm{rpm} ; n=10,9)$. i Maximal velocity reached on the rotarod was significantly higher for control than KD mice $(n=10,9)$. $\mathbf{j}-\mathbf{m} I O-C R F_{K D}$ and control mice were tested for anxiety-like behavior using the open field (OF) test. No differences in anxiety-like behavior were detected between $\mathrm{KD}$ and control mice $(n=9$, 9). Data are presented as mean \pm SEM. $\sim p<$ $0.07,{ }^{*} p<0.05,{ }^{* *} p<0.005$. Significant interaction (time $\times$ group) marked as ${ }^{\#} p<0.01$

f). Mice were then tested on the rotarod for three consecutive days. The rotating rod was accelerated by additional $10 \mathrm{rpm}$ each $\min (0-40 \mathrm{rpm}$ in $4 \mathrm{~min}), 4$ trials/day, for 2 days, followed by a third day on which the speed was accelerated by $20 \mathrm{rpm}$ per min (Fig. 2c; see Materials and methods). IO-CRF $\mathrm{KD}$ mice lasted shorter periods of time on the rotating rod, reaching significance only on day 3 (Fig. 2g). IO-CRF $\mathrm{KD}_{\mathrm{D}}$ reached lower maximal velocities in
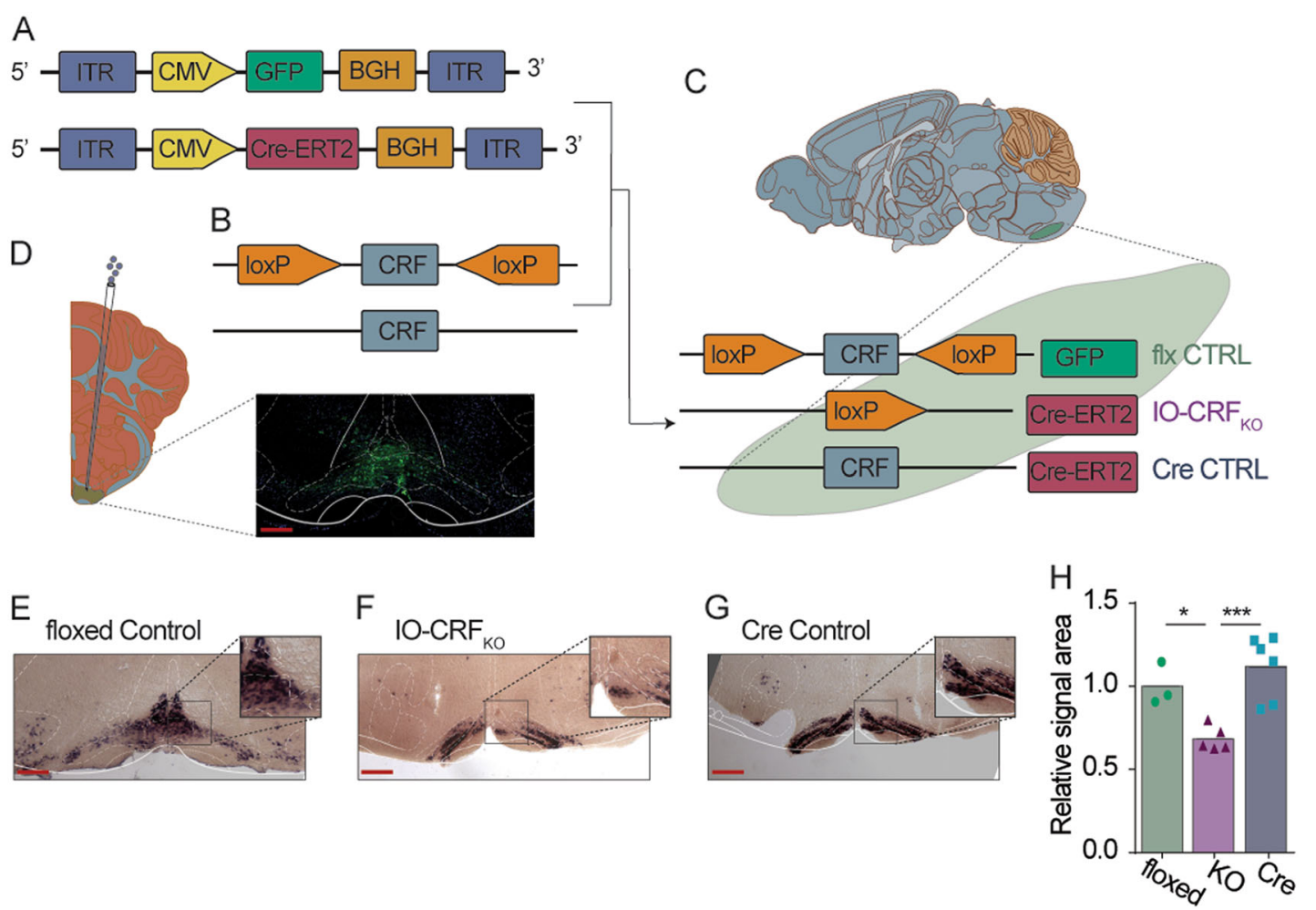

Fig. 3 Generation of IO-specific CRF partial KO mice. Floxed CRF or wild-type mice were injected with Cre expressing virus to induce a site-specific partial knockout (KO). a Schematic illustration of tamoxifen-induced Cre (Cre-ERT2) expressing adeno-associated virus and control GFP expressing virus. b, c Viral constructs were injected to floxed CRF mice and WT mice, to obtain control for floxed CRF background, site-specific partial CRF KO and control for the Cre activity. d Representative microscope image of virally infected IO of an adult mouse. Red scale bar $=250 \mu \mathrm{m}$. e- $\mathbf{g} \mathrm{In}$ situ hybridization using probes for CRF showing e the IO of floxed CRF mice injected with control virus (floxed control) and $\mathbf{f}$ with Cre-ERT2 virus (IO$\mathrm{CRF}_{\mathrm{KO}}$ ) and $\mathbf{g}$ WT littermates injected with Cre-ERT2 virus (Cre control). Red scale bar $=250 \mu \mathrm{m}$. $\mathbf{h}$ Relative signal area of CRF in situ hybridization obtained from floxed-control mice, partial IO-CRF $\mathrm{KO}$ and Cre-control (each dot represents an IO slice; $n=3,5,6$ slices for floxed control, KO and Cre control, respectively). Post hoc Fisher's LSD shows differences between IO-CRF KO group and the two control groups, but not between control groups. ${ }^{*} p<0.05,{ }^{* * *} p<0.005$ 

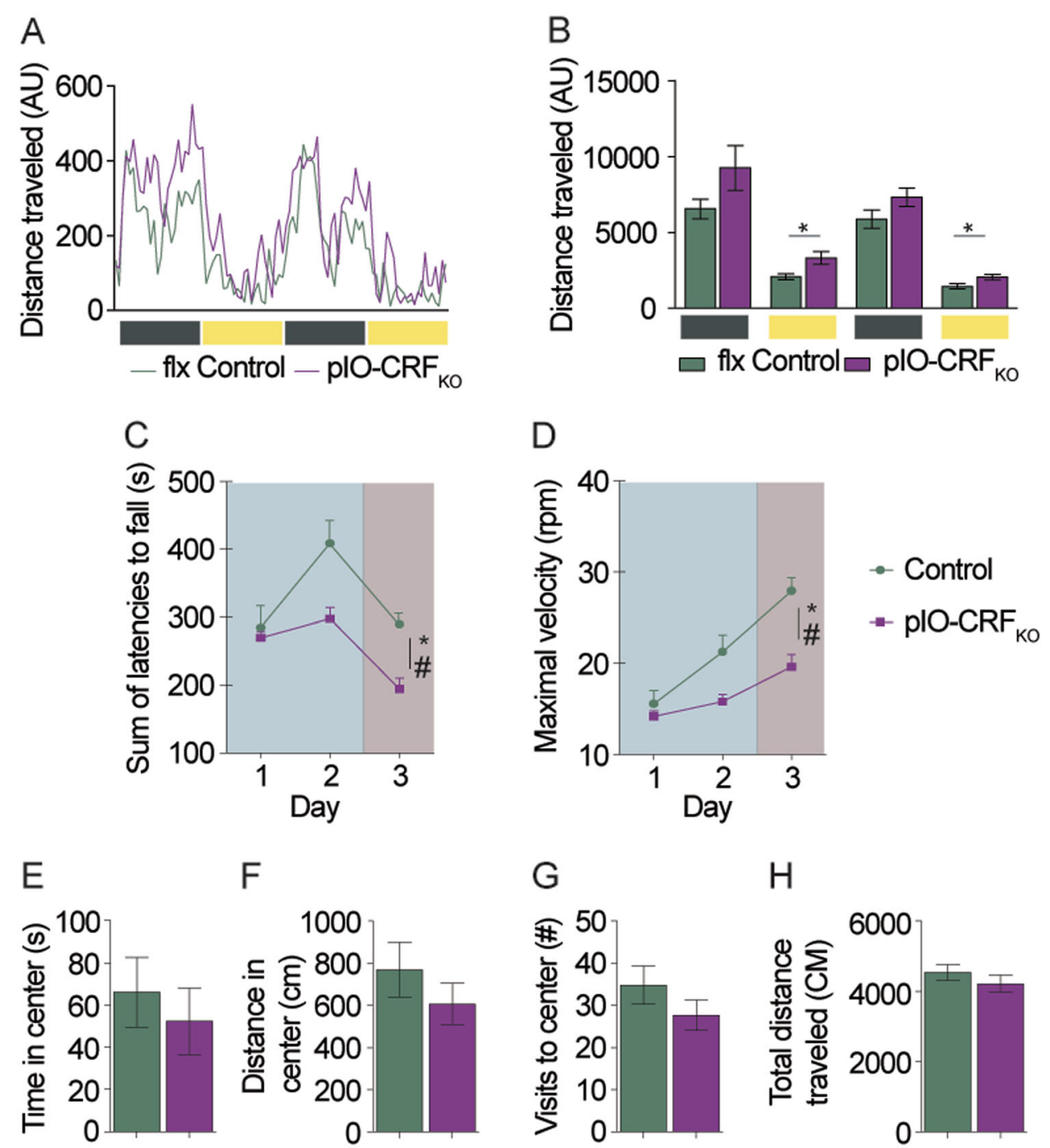

$\mathrm{F}$
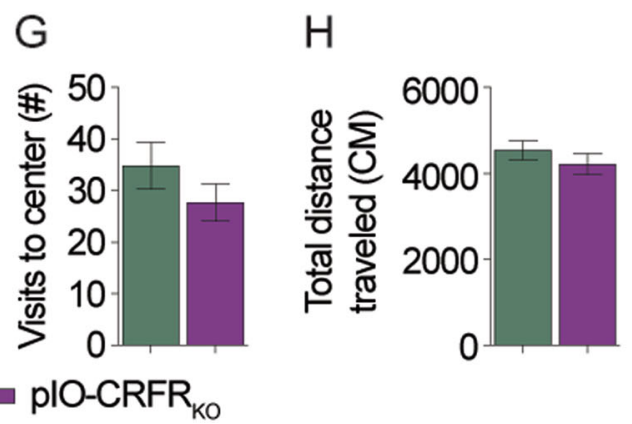

Fig. 4 IO-specific partial CRF-KO is sufficient to induce a motor deficit in mice. a, b Baseline motor activity of control and partial IO-CRF $F_{K O}(\mathrm{plO}-$ $\left.\mathrm{CRF}_{\mathrm{KO}}\right)$ mice. Groups did not differ in home cage locomotion during the dark phase, however plO-CRF $\mathrm{KO}_{\mathrm{KO}}$ mice had higher levels of locomotion during the light phase $(n=10,8)$. $\mathbf{c}, \mathbf{d}$ plO-CRF $\mathrm{KO}$ and control mice motor performance was tested using the rotarod. $\mathbf{c}$ IO-CRF KD were lower than control mice in sum of latencies to fall of the rotarod $(n=10,7)$. $\mathbf{d}$ plO-CRF $\mathrm{KO}$ also reached lower maximal velocities on the rotarod compared to controls $(n$ $=10,7)$. e-h plO-CRF KO and control mice were tested for anxiety-like behavior using the open field test. No differences in anxiety-like behavior were detected between $\mathrm{KO}$ and control mice $(n=10,8) .{ }^{*} p<0.05,{ }^{* * *} p<0.005$. Significant interaction (time $\times$ group) marked as ${ }^{\#} p<0.05$

the course of training (two-way repeated measures ANOVA, interaction of group and training day, $F_{(2,34)}=$ 6.303, $p=0.005, n=10,9$; Fig. 2h), Student's $t$-test reveals the difference between the groups becomes significant at the $20 \mathrm{rpm}$ speed $\left(t_{(17)}=2.698, p=0.015\right.$, Bonferronicorrected critical $p=0.0166$; Fig. 2e). In order to further assess baseline gait and coordination, we tested mice on the CatWalk (Noldus). Data were collected on the following measures: base of support (BOS), print position (to evaluate gait or balance disturbance), and Regularity Index (overall coordination assessment) (Supplementary
Fig. 2A-C). KD mice did not differ from control counterparts, implying that reduced IO-CRF levels are particularly relevant for motor tasks that require effort rather than for baseline locomotion (Supplementary Fig. 2B-C). When tested for anxiety-like behavior in the open-field (OF) test, IO-CRF $F_{\mathrm{KD}}$ mice did not differ from their control littermates, except for a tendency for significance in the number of visits to the center $(p=0.058$, Bonferroni correction critical $p=0.0125$; Fig. $2 \mathrm{j}-\mathrm{m}$ ). These results suggest that reduced IO-CRF can induce a specific challenge-induced motor phenotype. Next, we employed 
the Cre-lox KO system to induce a more pronounced IOCRF reduction.

\section{IO-specific partial CRF-KO is sufficient to induce motor deficit in mice}

Tamoxifen inducible $\mathrm{Cre}$ expressing adeno-associated virus (Cre- ERT2) or control virus was injected into adult floxed CRF mice (see Materials and Methods and Supplementary Methods; Fig. 4a, b). In order to insure Cre did not affect the cells' viability nor levels of CRF, WT littermates were also injected with Cre-ERT2 (Fig. 3a-c). All mice received tamoxifen solution by gavage (Fig. 3c). Location of the injection was validated using either GFP staining or staining against Cre (Fig. 3d). Post Cre induction, CRF levels were assessed using ISH on slices obtained from floxed mice injected with GFP expressing virus (floxed control; Fig. 4e), floxed mice injected with Cre-ERT2 (i.e., IO-CRF ${ }_{\mathrm{KO}}$; Fig. 3f) and WT mice injected with Cre-ERT2 (Cre control; Fig. 3g). Floxed CRF mice injected with Cre-ERT2 showed significantly less CRF ISH signal compared to both control groups (one-way ANOVA, $F_{(2,11)}=11.931, p=0.002$; Post hoc Fisher's LSD shows differences between IO-CRF $\mathrm{KO}_{\mathrm{KO}}$ group vs. Cre control group, $p=0.001$, and vs. floxed control group, $p$ $=0.014$; Fig. $3 \mathrm{~h}$ ). Importantly, the signal in slices obtained from Cre-ERT2 WT mice did not differ from that in floxed controls (Fig. 3h). Although CRF in the infected cells was completely abolished in IO-CRF $\mathrm{KO}_{\mathrm{KO}}$ mice, we were only able to cover a moderate part of the IO using intracerebral injections, and thus we obtained only a partial IO-CRF $\mathrm{KO}_{\mathrm{KO}}$ (pIO-CRF $\mathrm{CO}_{\mathrm{KO}} \sim 40 \%$ reduction; Fig. $3 \mathrm{~h}$ ).

Analysis of home-cage basal activity of $\mathrm{pIO}-\mathrm{CRF}_{\mathrm{KO}}$ mice and control mice revealed a small increase in locomotor activity of pIO-CRF $\mathrm{KO}_{\mathrm{KO}}$ mice compared to controls, only during the light phase $\left(t_{(16)}=3.120, p=0.007, t_{(16)}\right.$ $=3.05, p=0.008 ; n=10,8$, Bonferroni-corrected critical $p=0.0125$; Fig. 4a, b). In marked contrast, when the mice were tested on a challenging motor task, the rotarod, pIO$\mathrm{CRF}_{\mathrm{KO}}$ mice performed significantly worse than control mice (Fig. 4c, d). KO mice lasted shorter periods of time and showed a more gradual improvement during training (two-way repeated measures ANOVA, main effect for group, $F_{(1,15)}=6.235, p=0.025$; interaction $F_{(2,30)}=$ $3.715, p=0.036, n=10,7$; Fig. 4c) and reached lower maximal velocities (repeated measures ANOVA, main effect for group, $F_{(1,15)}=8.599, p=0.01$; interaction $F_{(2,30)}=6.169, p=0.006, n=10,7$; Fig. $\left.4 \mathrm{~d}\right)$. To further evaluate the gait and coordination of $\mathrm{pIO}-\mathrm{CRF}_{\mathrm{KO}}$ mice, they were tested on the CatWalk (Noldus; Supplementary Fig. 2A and 3). Mice with pIO-CRF $\mathrm{KO}_{\mathrm{O}}$ did not differ from controls in BOS, and in spite a difference in average print position, none of the differences yielded significance following multiple comparison $p$ value correction (Supplementary Fig. 3A). The Regulatory Index of KO mice did not differ from that of controls (Supplementary Fig. 3B). The $\mathrm{pIO}-\mathrm{CRF}_{\mathrm{KO}}$ group did not differ from controls in anxiety-like behavior as measured in the OF test (Fig. $4 \mathrm{e}-\mathrm{h}$ ). Taken together, IO-specific CRF manipulation reveals that IO-CRF plays an important role in challengeinduced motor tuning.

It has been repeatedly reported, mostly using immunostaining and electrophysiology, that $\mathrm{CRFR}_{1}$ is expressed in cerebellar $\mathrm{PCs}^{11,15,36-42}$. As the main output of the IO is to PCs, we hypothesized that the IO-CRF effect will be driven by PCs. However, we were unable to confirm $\mathrm{CRFR}_{1}$ expression in PCs of the CRFR1 reporter line $\left(\mathrm{CRFR}_{1}{ }^{\mathrm{GFP}}\right)$, using ISH for $\mathrm{CRFR}_{1}$ on sagittal mouse brain slices (Supplementary Fig. 4A-E). In contrast, with both methods a signal was detected in molecular layer interneurons (MLIs; red arrows), in the granular cell layer, and in the deep cerebellar nuclei (DCN) as expected (Supplementary Fig. 4B-E).

Next, we used PCP2 $^{\text {cre }}$ mice, which express Cre recombinase solely in PCs, and crossbred them with either floxed CRFR1 mice or floxed CRFR2 mice, (i.e., putative PC-CRFR $\left.1_{\mathrm{KO}} ; \mathrm{pPC}-\mathrm{CRFR} 2_{\mathrm{KO}}\right)$. We tested for levels of CRFR1 and CRFR2 mRNA in the cerebelli of this model but no differences were detected in CRFRs mRNA levels in pPC-CRFRs-KO (Supplementary Fig. 4F-G). $\mathrm{CRFR}_{1}$ immunohistochemistry on cerebelli from developmental CRFR ${ }_{1 K O}$ mice, on the pPC-CRFR ${ }_{1 K O}$ mice and WT mice demonstrates that the antibody gives a false signal in PCs. All these mouse lines showed a similar signal in PCs, which was inconsistent with the $\mathrm{CRFR}_{1}$-ISH signal and with $\mathrm{CRFR}_{1}{ }^{\mathrm{GFP}}$ reporter line GFP expression (as presented in Supplementary Fig. 4B-C, H).

To conclude, although IO-CRF clearly has a role in motor performance of the mouse, its site of action is not yet clear. We could not validate CRFR1 expression in the PCs of mice, the natural output for IO neurons, and this issue has been noted previously ${ }^{43-46}$. However, IO-CRF may affect MLIs via bulk transmission, or DCN neurons by CF collaterals as both have validated CRFR1 staining, or by an alternative CRFR on PCs (see Discussion).

\section{IO-CRF is "recruited" in stressful challenges that require motor activity}

Chronic stress, specifically CSDS, can induce long lasting motor impairments ${ }^{47,48}$. To study whether these long-term effects of CSDS correlate with changes in IOCRF, IO dissections from mice following CSDS were collected and analyzed for CRF mRNA levels using quantitative real-time PCR (qRT-PCR; Supplementary Methods; Fig. 5a). Following CSDS, the mice had an average of $50 \%$ lower IO-CRF mRNA levels compared to control mice $\left(t_{(11)}=3.252, p=0.008 ; n=8\right.$, 5; Fig. 5b). Importantly, cFos did not change in CSDS mice, implying there is no long-lasting change in IO activity in 

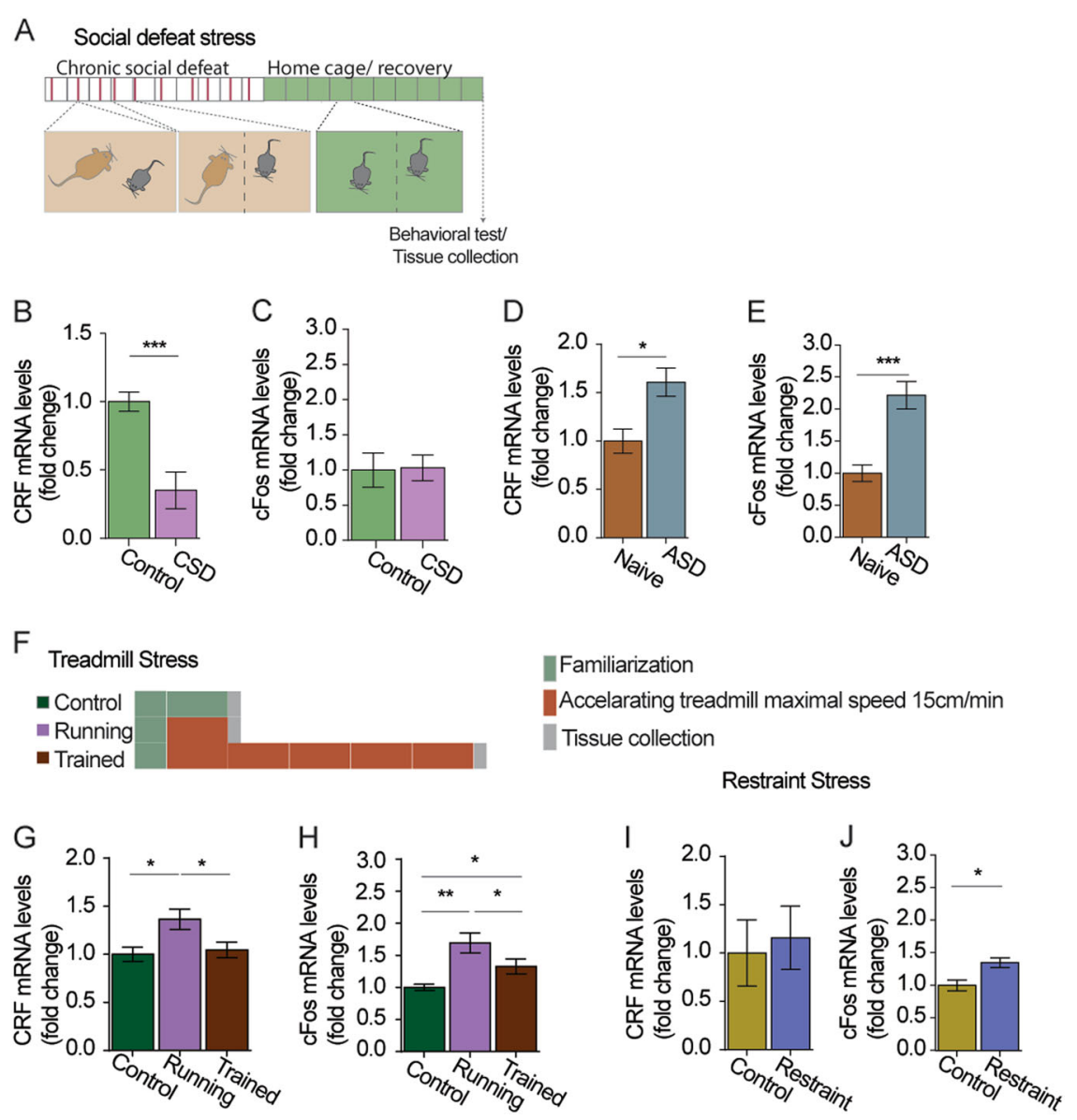

Fig. 5 Inferior olive CRF is "recruited" in stressful challenges that require motor activity. a Schematic illustration of the chronic social defeat stress (CSDS) paradigm. C57 mice were subjected to 5 min of physical contact with an aggressive ICR mouse (red line), followed by sensory contact for $24 \mathrm{~h}$, for 10 consecutive days following which mice were returned to a cage with their littermates for 10 days. b IO-CRF mRNA levels collected 10 days after the end of the CSDS protocol were reduced two-fold compared to controls $(n=8,5)$. $\mathbf{c}$ Long-lasting changes in $I O$ cFos levels were not detected in CSDS mice. $\mathbf{d}$ IO-CRF mRNA levels were increased 90 mins after single acute social stress (ASD) compare to that of control mice $(n=8,7)$. e An increase in $1 O$ cFos mRNA levels was also apparent in ASD mice indicating increased IO activity during the defeat $(n=8,7)$. f Schematic illustration of the treadmill protocol used to induce "forced flight response". Mice were divided into three groups; all groups were familiarized with the apparatus and the shocker 1 day prior to the start of their protocol (green rectangle). One group was trained on the treadmill at a different acceleration pace but similar maximal speed (15 m/min) for 20 min for 4 days ("trained"; brown rectangles). The control group went on the treadmill with the shocker active, but without the treadmill belt moving (i.e., mice did not need to run in order to avoid the shock; "passive avoidance" = "control" group), and the last group was faced with the need to run away from the shocker on test day for the first time ("running" group; single brown rectangle). $\mathbf{g}$ IO-CRF mRNA levels 90 min after the beginning of the run on an accelerating treadmill were increased only in the "running" group compared to the "trained" and to the "control" group ( $n=6,8,8$ for "Control", "Running", and "Trained", respectively). $\mathbf{h}$ Following treadmill run, cFos mRNA levels differed between all groups. The "running" group showed the highest average cFos mRNA levels, followed by the "trained" group ( $n=6,8,8$ for "control", "running", and "trained", respectively). i IO-CRF mRNA levels 90 min following the initiation of a single acute restraint stress do not differ from that of control mice $(n=5,5)$. j cFos levels in the $1 O$ were mildly increased following acute restraint stress $(n=5,4)$. Data are presented in mean \pm SEM. ${ }^{*} p<0.05,{ }^{* *} p<0.01,{ }^{* *} p<0.001$

chronically stressed mice compared to control mice (Fig. 5c). A different set of mice following a similar CSDS endured less time on the rotarod (Supplementary Fig. 5A) and reached lower maximal velocities (Supplementary Fig. 5B) as reported previously ${ }^{48}$.

The marked long-lasting downregulation of IO-CRF following CSDS raised the hypothesis that during the social defeat, IO-CRF is "recruited" to cope with the challenge. To test this, we exposed C57/BL mice to a single social defeat encounter (acute social defeat, ASD). Measuring mRNA changes shortly after a challenge allows us to detect short-term changes in transcription. These changes are likely a result of restoration of neuropeptide levels following its release. As hypothesized, IO-CRF mRNA was significantly increased shortly following the ASD (Mann-Whitney $U=8, p=0.021, n=8$, 7; Fig. 5a). To evaluate IO neuronal activity in response to this paradigm, we assessed cFos mRNA levels as well. ASD 
mice had a significant increase in cFos mRNA $\left(t_{(13)}=\right.$ 4.784, $p<0.001 ; n=8$, 7; Fig. 5b).

We further hypothesized that IO-CRF is recruited specifically in stressful challenges that require a well-tuned motor response. We used the accelerating treadmill apparatus to induce a "forced flight response" in the mice, which represents a stressful task that is also inherently motor. Mice were habituated to the treadmill apparatus for $10 \mathrm{~min}$ on day 1 while no running was required (Supplementary Methods; Fig. 5c). On the following days, mice were divided into three groups: "control" group was in the apparatus but not running, "trained" group was trained for total of 5 days on the treadmill, and "running" group ran for the first time on test/tissue collection day (Fig. 5c). CRF mRNA showed a marked increase in the "running" group, but not in the "trained" group, compared to controls (one-way ANOVA, $F_{(2,19)}=4.206, p=0.031$, post hoc Fisher's LSD running-ctrl $p=0.021$, trainedrunning $p=0.025 ; n=6,8,8$ for "control", "running" and "trained", respectively; Fig. 5d). Since CRF mRNA was increased in the "running" group only, it suggests it is independent from IO activity per se, as cFos was increased in both the "running" and "trained" groups compared to "control" (one-way ANOVA, $F_{(2,19)}=9.684, p<0.001$, post hoc Fisher's LSD, control-running $p<0.001$, controltrained $p=0.044$, running-trained $p=0.026 ; n=6,8,8$ for "control", "running", and "trained", respectively; Fig. $5 e)$.

Finally, we used restraint stress to determine if a "nonmotor" stressful and novel situation results in IO-CRF mRNA regulation. Mice were subjected to immobilization stress shortly followed by tissue collection (see Supplementary Methods). Control mice were left in their homecages. IO-CRF mRNA did not differ between stressed and control mice, although a small but significant increase in IO cFos was observed $\left(t_{(7)}=3.215, p=0.015, n=5\right.$, 4; Fig. $5 \mathrm{f}, \mathrm{g})$. Taken together, these results suggest that IO-CRF is important for specific stress induced responses that allow for "motor coping", but not for all stressful situations.

In this study, we have shown that CRF is expressed throughout the IO and expressed in the majority of IO neurons. Using site-specific KD and partial $\mathrm{KO}$ in adult mice, we found reduced levels of CRF resulted in maladaptive motor performance. Lastly, we presented evidence of regulation on IO CRF under specific conditions. This study implicates IO-CRF in the complete adaptive stress response.

\section{Discussion}

In the current study, we examined the role of IO-CRF in mediating stress related motor performance under challenging conditions. In agreement with previous reports, we showed that CRF is highly expressed in the IO and CFs rising from the IO in mice and humans ${ }^{10,11,14,15,17,49}$. The fact that CRF is expressed in the majority IO neurons indicates their importance. However, to date, no study has examined the role of IO neurons in behavior or physiology.

Here we showed that mice with reduced IO-CRF levels presented decreased motor abilities when tested on the rotarod. These experiments indicate that IO-CRF plays a role in the motor capabilities of mice, when faced with a challenge, but does not affect general basal locomotion. Our results are similar to a very recent publication manipulating IO-CRF in rats ${ }^{50}$. Wang and colleagues show that KD rats present lower performance on the rotarod, as well as having some gait disturbance. Our gait analysis results did not yield significant differences (Supplementary Figures 2B and 3A). Nevertheless, on average, pIO-CRFKO showed differences in gait, in a similar direction to those presented in the work by Wang et al. (Supplementary Fig. 3A) ${ }^{50}$. A likely explanation is that the extent of our $\mathrm{KD}$ and $\mathrm{KO}$ was somewhat smaller, and thus our behavioral effect somewhat milder. Interestingly low to moderate levels of anxiety or CRF in certain brain regions seem to lead to increased locomotion ${ }^{1}$. In our work, a mild increase in locomotion was detected only in the partial IO-CRF $\mathrm{KO}_{\mathrm{O}}$ model (in accordance with "mild stress" situations), which may imply that a strong CRF manipulation may affect locomotion. However further analysis is required in order to conclusively determine a causative link between IO-CRF and locomotion, as it was not detected in the KD mouse model. Nevertheless, it is important to note that generally IO plays a particular role in motor learning and adjustment of movements when facing variable challenges, more so than in baseline locomotion $^{51,52}$. Importantly, IO-CRF manipulation did not affect stress-related behaviors in mice, emphasizing a purely motor role.

Previously published work has established that chronic stress, in particular CSDS, disrupts motor abilities in rodents $^{47,48,53}$. Human studies with PTSD patients revealed that cerebellar connectivity is altered in patients compared to control subjects ${ }^{20}$. Environmental factors linked with depression (including post-stroke depression) were also found to be related to cerebellar connectivity ${ }^{54}$. Cerebellar connectivity was implemented in social anxiety and chronic work stress ${ }^{22,55}$. Moreover, co-morbidity of cerebellar ataxia and depression might involve CRF disfunction $^{56-58}$. However, the direction of the relationship between stress and motor capabilities is not yet clear, and thus further research is needed ${ }^{21,58}$. In our study, we found that defeated mice have a persistent downregulation of IO-CRF mRNA. This implies that maladaptive emotional states are correlated with changes in cerebellar circuitry, which in turn may affect motor related skills, as demonstrated in mice motor performance following $\operatorname{CSDS}^{53}$ (Supplementary Fig. 5). 
CRF transcript was previously shown to be regulated by different challenges in several brain areas. Moreover, IOCRF mRNA was specifically shown to be differentially regulated in ataxic mouse lines compared to controls and following harmaline induced tremor and other ataxia models ${ }^{1,27,59-64}$. We further showed that IO CRF mRNA is specifically upregulated shortly after stressful situations that require motor coping. In light of these findings, we hypothesize that IO-CRF is "recruited" specifically when an "enhancement" in coordination/motor capacity is needed in order to efficiently cope with a challenge or stressor.

This hypothesis supports the concept of an upstream mechanism directing different "stress pathways", as previously suggested ${ }^{65,66}$. Thus far, several brain structures, such as the central amygdala and the periaqueductal gray, have shown differential internal activation that determines whether the behavioral outcome is freezing of fleeting. The differential internal activity in these structures depends, amongst other things, on whether the situation allows for flight or promotes freezing (e.g., if there is no flight route ${ }^{66,67}$ ). Whether the periaqueductal gray or other structures can regulate CRF release from the IO would be an interesting question to explore in the future.

CFs constitute a direct pathway from the IO to PCs in the cerebellar cortex. The effect of CRF on PCs' electrophysiological in vitro activity and development have been previously reported ${ }^{39,40,68-70}$, however, the expression of CRFR1 in PCs has also been challenged ${ }^{43-45}$. In spite of the canonical statue of IO-PC pathway, it is important to note that CRFR1 has been reported to be expressed in MLIs and DCN as well ${ }^{44,71}$. These pathways (IO to MLIs, IO to DCNs) are additional plausible pathways through which IO-CRF may affect behavior ${ }^{50,72,73}$. Importantly, a recent publication by Wang et al., indeed shows that CRF injection to the DCN ameliorates IO-CRFKD induced motor phenotype ${ }^{50}$. Besides, MLIs, although traditionally affected by granular cells' parallel fibers, do not have any other likely input of CRF except for the IO. This further supports the likelihood of IO-CRF affecting via bulk transmission ${ }^{74}$. Future research should examine the effect of CRFR1 manipulations in the various cell types likely to be affected by CRF. Moreover, genetic studies including single cell sequencing are needed to further assess the expression of CRFRs in PCs, and their extent. To conclude, IO-CRF participates in the IO-cerebellar pathway and affects the organisms' motor capabilities under specific conditions. We are yet to determine what the upstream and downstream mechanisms involved are, as well as the cellular mechanisms involved.

It is important to note the human aspect, as CRF is highly expressed in the human IO, and PTSD and stress related pathology patients show altered cerebellar connectivity ${ }^{20-22,55}$. Therefore, it is essential to consider and further explore if chronic stress has an impact on the ability to physically cope with challenging situations. This aspect has been mostly disregarded in the field of stressneurobiology, despite basic central motor mechanisms being as important as emotional mechanisms in an organism's survival.

\section{Acknowledgements}

We thank Sharon Ovadia for his devoted assistance with animal care. We thank Jessica Keverne for her professional English editing, formatting and scientific input. A.C. is Head of the Max Planck Society-Weizmann Institute of Science Laboratory for Experimental Neuropsychiatry and Behavioral Neurogenetics. A. C.'s work is supported by an FP7 grant from the European Research Council (260463); a research grant from the Israel Science Foundation (1565/15); the ERANET Program, supported by the Chief Scientist Office of the Israeli Ministry of Health (3-11389, A.C.); the Federal Ministry of Education and Research under the funding code 01KU1501A; the I-CORE Program of the Planning and Budgeting Committee; and the Israel Science Foundation (grant no. 1916/12). A.C. also receives research support from Roberto and Renata Ruhman; the Nella and Leon Benoziyo Center for Neurological Diseases; the Henry Chanoch Krenter Institute for Biomedical Imaging and Genomics; the Perlman Family Foundation, founded by Louis L. and Anita M. Perlman; the Adelis Foundation and the Irving I. Moskowitz Foundation; and the Marc Besen and the Pratt Foundation.

\section{Author details}

'Department of Neurobiology, Weizmann Institute of Science, Rehovot 76100, Israel. ${ }^{2}$ Department of Stress Neurobiology and Neurogenetics, Max Planck Institute of Psychiatry, 80804 Munich, Germany. ${ }^{3}$ Department of Veterinary Resources, Weizmann Institute of Science, 76100 Rehovot, Israel

Conflict of interest

The authors declare that they have no conflict of interest.

\section{Publisher's note}

Springer Nature remains neutral with regard to jurisdictional claims in published maps and institutional affiliations.

Supplementary Information accompanies this paper at https://doi.org/ 10.1038/s41398-018-0145-3.

Received: 5 July 2017 Revised: 5 February 2018 Accepted: 20 February 2018 Published online: 25 May 2018

\section{References}

1. Sztainberg, Y. \& Chen, A. in Handbook of Neuroendocrinology (eds Fink, G., Pfaff, D. W. \& Levine, J. E.) Ch. 15, pp. 355-375 (Academic Press, San Diego, 2017).

2. Henckens, M. J. A. G., Deussing, J. M. \& Chen, A. Region-specific roles of the corticotropin-releasing factor-urocortin system in stress. Nat. Rev. Neurosci. 17, 636-651 (2016)

3. Hauger, R. L., Risbrough, V., Oakley, R. H., Olivares-Reyes, J. A. \& Dautzenberg, F. $M$. Role of CRF receptor signaling in stress vulnerability, anxiety, and depression. Ann. N. Y. Acad. Sci. 1179, 120-143 (2009).

4. Heinrichs, S. C. \& Koob, G. F. Corticotropin-releasing factor in brain: a role in activation, arousal, and affect regulation. J. Pharmacol. Exp. Ther. 311, 427-440 (2004).

5. Koob, G. F. \& Heinrichs, S. C. A role for corticotropin releasing factor and urocortin in behavioral responses to stressors. Brain Res. 848, 141-152 (1999).

6. Olschowka, J. A., O'Donohue, T. L., Mueller, G. P. \& Jacobowitz, D. M. The distribution of corticotropin releasing factor-like immunoreactive neurons in rat brain. Peptides 3, 995-1015 (1982).

7. Merchenthaler, I. Corticotropin releasing factor (CRF)-like immunoreactivity in the rat central nervous system. Extrahypothalamic distribution. Peptides 5, 53-69 (1984). 
8. Skofitsch, G., Insel, T. R. \& Jacobowitz, D. M. Binding sites for corticotropin releasing factor in sensory areas of the rat hindbrain and spinal cord. Brain Res. Bull. 15, 519-522 (1985).

9. Palkovits, M., Léránth, C., Görcs, T. \& Young, W. S. Corticotropin-releasing factor in the olivocerebellar tract of rats: demonstration by light- and electronmicroscopic immunohistochemistry and in situ hybridization histochemistry. Proc. Natl Acad. Sci. USA 84, 3911-3915 (1987)

10. Cha, C. I. \& Foote, S. L. Corticotropin-releasing factor in olivocerebellar climbing-fiber system of monkey (Saimiri sciureus and Macaca fascicularis): parasagittal and regional organization visualized by immunohistochemistry. J. Neurosci. J. Soc. Neurosci. 8, 4121-4137 (1988).

11. Cummings, S., Sharp, B. \& Elde, R. Corticotropin-releasing factor in cerebellar afferent systems: a combined immunohistochemistry and retrograde transport study. J. Neurosci. J. Soc. Neurosci. 8, 543-554 (1988).

12. Foote, S. L. \& Cha, C. I. Distribution of corticotropin-releasing-factor-like immunoreactivity in brainstem of two monkey species (Saimiri sciureus and Macaca fascicularis): an immunohistochemical study. J. Comp. Neurol. 276, 239-264 (1988)

13. Kitahama, K. et al. Localization of CRF-immunoreactive neurons in the cat medulla oblongata: their presence in the inferior olive. Cell. Tissue Res. 251, 137-143 (1988).

14. Errico, P. \& Barmack, N. H. Origins of cerebellar mossy and climbing fibers immunoreactive for corticotropin-releasing factor in the rabbit. J. Comp. Neurol. 336, 307-320 (1993).

15. King, J. S., Madtes, P., Bishop, G. A. \& Overbeck, T. L. The distribution of corticotropin-releasing factor (CRF), CRF binding sites and CRF1 receptor mRNA in the mouse cerebellum. Prog. Brain Res. 114, 55-66 (1997).

16. Drolet, G. \& Rivest, S. Corticotropin-releasing hormone and its receptors; an evaluation at the transcription level in vivo. Peptides 22, 761-767 (2001).

17. Sawada, K., Fukui, Y. \& Hawkes, R. Spatial distribution of corticotropin-releasing factor immunopositive climbing fibers in the mouse cerebellum: analysis by whole mount immunohistochemistry. Brain Res. 1222, 106-117 (2008).

18. Mauk, M. D., Medina, J. F., Nores, W. L. \& Ohyama, T. Cerebellar function: coordination, learning or timing? Curr. Biol. 10, R522-R525 (2000).

19. Ohyama, T., Nores, W. L., Murphy, M. \& Mauk, M. D. What the cerebellum computes. Trends Neurosci. 26, 222-227 (2003).

20. Thome, J. et al. Desynchronization of autonomic response and central autonomic network connectivity in posttraumatic stress disorder. Hum. Brain Mapp. 38, 27-40 (2017).

21. Nieuwenhuys, A. \& Oudejans, R. R. D. Anxiety and perceptual-motor performance: toward an integrated model of concepts, mechanisms, and processes Psychol. Res. 76, 747-759 (2012).

22. Yuan, M. et al. Group cognitive behavioral therapy modulates the resting-state functional connectivity of amygdala-related network in patients with generalized social anxiety disorder. BMC Psychiatry 16, 198 (2016).

23. Gupta, M. A. Review of somatic symptoms in post-traumatic stress disorder. Int. Rev. Psychiatry 25, 86-99 (2013).

24. Volk, N. et al. Amygdalar microRNA-15a is essential for coping with chronic stress. Cell Rep. 17, 1882-1891 (2016).

25. Neufeld-Cohen, A. et al. A triple urocortin knockout mouse model reveals an essential role for urocortins in stress recovery. Proc. Natl Acad. Sci. USA 107, 19020-19025 (2010).

26. Ramot, A. et al. Hypothalamic CRFR1 is essential for HPA axis regulation following chronic stress. Nat. Neurosci. 20, 385-388 (2017).

27. Elliott, E., Ezra-Nevo, G., Regev, L., Neufeld-Cohen, A. \& Chen, A. Resilience to social stress coincides with functional DNA methylation of the Crf gene in adult mice. Nat. Neurosci. 13, 1351-1353 (2010).

28. Regev, L., Tsoory, M., Gil, S. \& Chen, A. Site-specific genetic manipulation of amygdala corticotropin-releasing factor reveals its imperative role in mediating behavioral response to challenge. Biol. Psychiatry 71, 317-326 (2012).

29. Chen, A. et al. Urocortin 2-deficient mice exhibit gender-specific alterations in circadian hypothalamus-pituitary-adrenal axis and depressive-like behavior. J. Neurosci. J. Soc. Neurosci. 26, 5500-5510 (2006).

30. Kuperman, Y. et al. CRFR1 in AgRP neurons modulates sympathetic nervous system activity to adapt to cold stress and fasting. Cell Metab. 23, 1185-1199 (2016).

31. Ye, L. et al. Wiring and molecular features of prefrontal ensembles representing distinct experiences. Cell 165, 1776-1788 (2016).

32. Regev, L. et al. Prolonged and site-specific over-expression of corticotropinreleasing factor reveals differential roles for extended amygdala nuclei in emotional regulation. Mol. Psychiatry 16, 714-728 (2011).
33. Taniguchi, $\mathrm{H}$. et al. A resource of Cre driver lines for genetic targeting of GABAergic neurons in cerebral cortex. Neuron 71, 995-1013 (2011).

34. Madisen, L. et al. A robust and high-throughput Cre reporting and characterization system for the whole mouse brain. Nat. Neurosci. 13, 133-140 (2010).

35. Hawrylycz, M. J. et al. An anatomically comprehensive atlas of the adult human brain transcriptome. Nature 489, 391-399 (2012).

36. Bishop, G. A., Seelandt, C. M. \& King, J. S. Cellular localization of corticotropin releasing factor receptors in the adult mouse cerebellum. Neuroscience 101 1083-1092 (2000).

37. Bishop, G. A. Neuromodulatory effects of corticotropin releasing factor on cerebellar Purkinje cells: an in vivo study in the cat. Neuroscience 39, 251-257 (1990).

38. King, J. S. \& Bishop, G. A. Localization of the type 1 corticotropin releasing factor receptor (CRF-R1) in the embryonic mouse cerebellum. J. Neurocytol. 32 305-316 (2003).

39. Miyata, M., Okada, D., Hashimoto, K., Kano, M. \& Ito, M. Corticotropin-releasing factor plays a permissive role in cerebellar long-term depression. Neuron 22, 763-775 (1999).

40. Schmolesky, M. T., De Ruiter, M. M., De Zeeuw, C. I. \& Hansel, C. The neuropeptide corticotropin-releasing factor regulates excitatory transmission and plasticity at the climbing fibre-Purkinje cell synapse. Eur. J. Neurosci. 25, 1460-1466 (2007).

41. Tian, J.-B., King, J. S. \& Bishop, G. A. Stimulation of the inferior olivary complex alters the distribution of the type 1 corticotropin releasing factor receptor in the adult rat cerebellar cortex. Neuroscience 153, 308-317 (2008).

42. Chen, Y., Brunson, K. L., Müller, M. B., Cariaga, W. \& Baram, T. Z. Immunocytochemical distribution of corticotropin-releasing hormone receptor type-1 (CRF(1))-like immunoreactivity in the mouse brain: light microscopy analysis using an antibody directed against the C-terminus. J. Comp. Neurol. 420, 305-323 (2000).

43. Refojo, D. et al. Glutamatergic and dopaminergic neurons mediate anxiogenic and anxiolytic effects of CRHR1. Science 333, 1903-1907 (2011).

44. Justice, N. J., Yuan, Z. F., Sawchenko, P. E. \& Vale, W. Type 1 corticotropinreleasing factor receptor expression reported in BAC transgenic mice: implications for reconciling ligand-receptor mismatch in the central corticotropinreleasing factor system. J. Comp. Neurol. 511, 479-496 (2008).

45. Sánchez, M. M., Young, L. J., Plotsky, P. M. \& Insel, T. R. Autoradiographic and in situ hybridization localization of corticotropin-releasing factor 1 and 2 receptors in nonhuman primate brain. J. Comp. Neurol. 408, 365-377 (1999).

46. Allen Institute for Brain Science. Allen Mouse Brain Atlas. http://mouse.brainmap.org (2015).

47. Mizoguchi, K., Yuzurihara, M., Ishige, A., Sasaki, H. \& Tabira, T. Chronic stress impairs rotarod performance in rats: implications for depressive state. Pharmacol. Biochem. Behav. 71, 79-84 (2002).

48. Tomas-Roig, J. et al. Social defeat leads to changes in the endocannabinoid system: an overexpression of calreticulin and motor impairment in mice. Behav. Brain Res. 303, 34-43 (2016).

49. Chang, D., Yi, S. J. \& Baram, T. Z. Developmental profile of corticotropin releasing hormone messenger RNA in the rat inferior olive. Int J. Dev. Neurosci. J. Int Soc. Dev. Neurosci. 14,69-76 (1996).

50. Wang, Y. et al. Role of corticotropin-releasing factor in cerebellar motor control and ataxia. Curr. Biol. 27, 2661-2669.e5 (2017).

51. De Zeeuw, C. I. et al. Microcircuitry and function of the inferior olive. Trends Neurosci. 21, 391-400 (1998).

52. Sillitoe, R. V. An introduction to journal club in the Cerebellum. Cerebellum 11, 828 (2012).

53. Metz, G. A., Schwab, M. E. \& Welzl, H. The effects of acute and chronic stress on motor and sensory performance in male Lewis rats. Physiol. Behav. 72, 29-35 (2001).

54. Córdova-Palomera, A. et al. Environmental factors linked to depression vulnerability are associated with altered cerebellar resting-state synchronization. Sci. Rep. 6, 37384 (2016).

55. Golkar, A. et al. The influence of work-related chronic stress on the regulation of emotion and on functional connectivity in the brain. PLOS ONE 9, e104550 (2014).

56. Lo, R. Y. et al. Depression and clinical progression in spinocerebellar ataxias. Park. Relat. Disord. 22, 87-92 (2016).

57. Schmitz-Hübsch, T. et al. Depression comorbidity in spinocerebellar ataxia. Mov. Disord. 26, 870-876 (2011). 
58. Suemaru, S. et al. Cerebrospinal fluid corticotropin-releasing hormone in neurodegenerative diseases: reduction in spinocerebellar degeneration. Life. Sci. 57, 2231-2235 (1995).

59. Sawada, K. et al. Over-expression of corticotropin-releasing factor mRNA in inferior olivary neurons of rolling mouse Nagoya. Brain Res. Mol. Brain Res. 117, 190-195 (2003).

60. Cummings, S., Hinds, D. \& Young, W. S. Corticotropin-releasing factor mRNA increases in the inferior olivary complex during harmaline-induced tremor. Brain Res. 660, 199-208 (1994).

61. Ivy, A. S. et al. Hippocampal dysfunction and cognitive impairments provoked by chronic early-life stress involve excessive activation of $\mathrm{CRH}$ receptors. J. Neurosci. J. Soc. Neurosci. 30, 13005-13015 (2010).

62. O'Malley, D., Dinan, T. G. \& Cryan, J. F. Neonatal maternal separation in the rat impacts on the stress responsivity of central corticotropin-releasing factor receptors in adulthood. Psychopharmacology 214, 221-229 (2011).

63. Hauger, R. L., Risbrough, V., Brauns, O. \& Dautzenberg, F. M. Corticotropin releasing factor (CRF) receptor signaling in the central nervous system: new molecular targets. CNS Neurol. Disord. Drug. Targets 5, 453-479 (2006).

64. Jeong, Y. G. et al. Corticotropin-releasing factor immunoreactivity increases in the cerebellar climbing fibers in the novel ataxic mutant mouse, Pogo. Anat. Histol. Embryol. 35, 111-115 (2006).

65. Joëls, M. \& Baram, T. Z. The neuro-symphony of stress. Nat. Rev. Neurosci. 10, 459-466 (2009).

66. Fadok, J. P. et al. A competitive inhibitory circuit for selection of active and passive fear responses. Nature 542, 96-100 (2017).
67. Farook, J. M., Wang, Q., Moochhala, S. M., Zhu, Z. Y., Lee, L. \& Wong, P. T. H. Distinct regions of periaqueductal gray (PAG) are involved in freezing behavior in hooded PVG rats on the cat-freezing test apparatus. Neurosci. Lett. 354, 139-142 (2004)

68. Swinny, J. D. et al. Corticotropin-releasing factor and urocortin differentially modulate rat Purkinje cell dendritic outgrowth and differentiation in vitro. Eur. J. Neurosci. 19, 1749-1758 (2004).

69. Libster, A. M., Title, B. \& Yarom, Y. Corticotropin-releasing factor increases Purkinje neuron excitability by modulating sodium, potassium, and Ih currents. J. Neurophysiol. 114, 3339-3350 (2015).

70. Bishop, G. A. Development of a corticotropin-releasing factor-mediated effect on the firing rate of Purkinje cells in the postnatal mouse cerebellum. Exp. Neurol. 178, 165-174 (2002).

71. Van Pett, K. et al. Distribution of mRNAs encoding CRF receptors in brain and pituitary of rat and mouse. J. Comp. Neurol. 428, 191-212 (2000).

72. Apps, R. \& Garwicz, M. Anatomical and physiological foundations of cerebellar information processing. Nat. Rev. Neurosci. 6, 297-311 (2005).

73. D'Angelo E. The Organization of Plasticity in the Cerebellar Cortex: From Synapses to Control. In: Progress in Brain Research (Cerebellar Learning; vol. 210) (ed. Ramnani, N.) Ch. 2, pp. 31-58 (Elsevier, Amsterdam, Netherlands, 2010). http://www.sciencedirect.com/science/ article/pii/B9780444633569000029

74. Szapiro, G. \& Barbour, B. Multiple climbing fibers signal to molecular layer interneurons exclusively via glutamate spillover. Nat. Neurosci. 10, 735-742 (2007). 